\title{
Revealing Nanoscale Order and Strain in Lightweight FeMnAIC Steel Alloys with Atomic-resolution STEM
}

Michael Xu, Abinash Kumar and James LeBeau

Massachusetts Institute of Technology, Cambridge, Massachusetts, United States

High-Mn Fe-Mn-Al-C steels are lightweight alloys that have been shown to exhibit relatively high strength in addition to high ductility, properties unique with respect to those of many other classes of steels [1,2]. One of the mechanisms linked to enhanced strain-hardening and ductility is short-range order (SRO)-linked planar glide [3]. As a result, there is much interest in characterizing the nanoscale ordering of alloys having these enhanced mechanical properties [4]. Using conventional transmission electron microscopy, the nanoscale structure of FeMnAlC alloys has recently been shown to be non-homogenous with ordered clusters in an austenite matrix [5], yet, direct observation of these ordered regions and the local strain fields that influence properties remain.

In this presentation, we will show that direct observation of short-range ordering in the Fe-28Mn-9Al-0.8C lightweight steel is possible using HAADF STEM. To characterize the extent of ordering, we will highlight the use of atom column intensity correlation analysis of the high SNR Revolving STEM images [4, 6]. For example, Figure 1a shows a low-magnification LAADF STEM image of the FeMnAlC alloy in which a series of dislocations is seen. First, in order to determine the presence of intrinsic ordering in the bulk, atomicresolution imaging and analysis is performed away from these defects. Figure $1 \mathrm{~b}$ shows an atomic resolution HAADF RevSTEM image, from which near neighbor atom column intensity ratios are calculated. As shown in Figures $2 \mathrm{a}$ and $2 \mathrm{~b}$, intensity and correlation analysis, respectively, reveals regions of strongly correlated order separated by anti-phase boundaries. These correlated atom column intensities point to the presence of an ordered $\mathrm{L}_{2}$ structure composed of $\mathrm{Fe} / \mathrm{Mn}$ and $\mathrm{Al}$.

We will also demonstrate that 4D STEM imaging, coupled with drift-corrected annular dark field and integrated differential phase contrast imaging (iDPC), offers new insights into the structure of FeMnAlC lightweight steels. Using the aforementioned methods, we will discuss the effect of heat treatments on the formation of local atomic ordering in these lightweight steels and the modulation of strain fields at and near defects such as twin boundaries and dislocations (Figure 1a). Finally, we will compare our results with those obtained by conventional TEM methods to provide a connection to the long-range microstructure in FeMnAlC alloys [7].

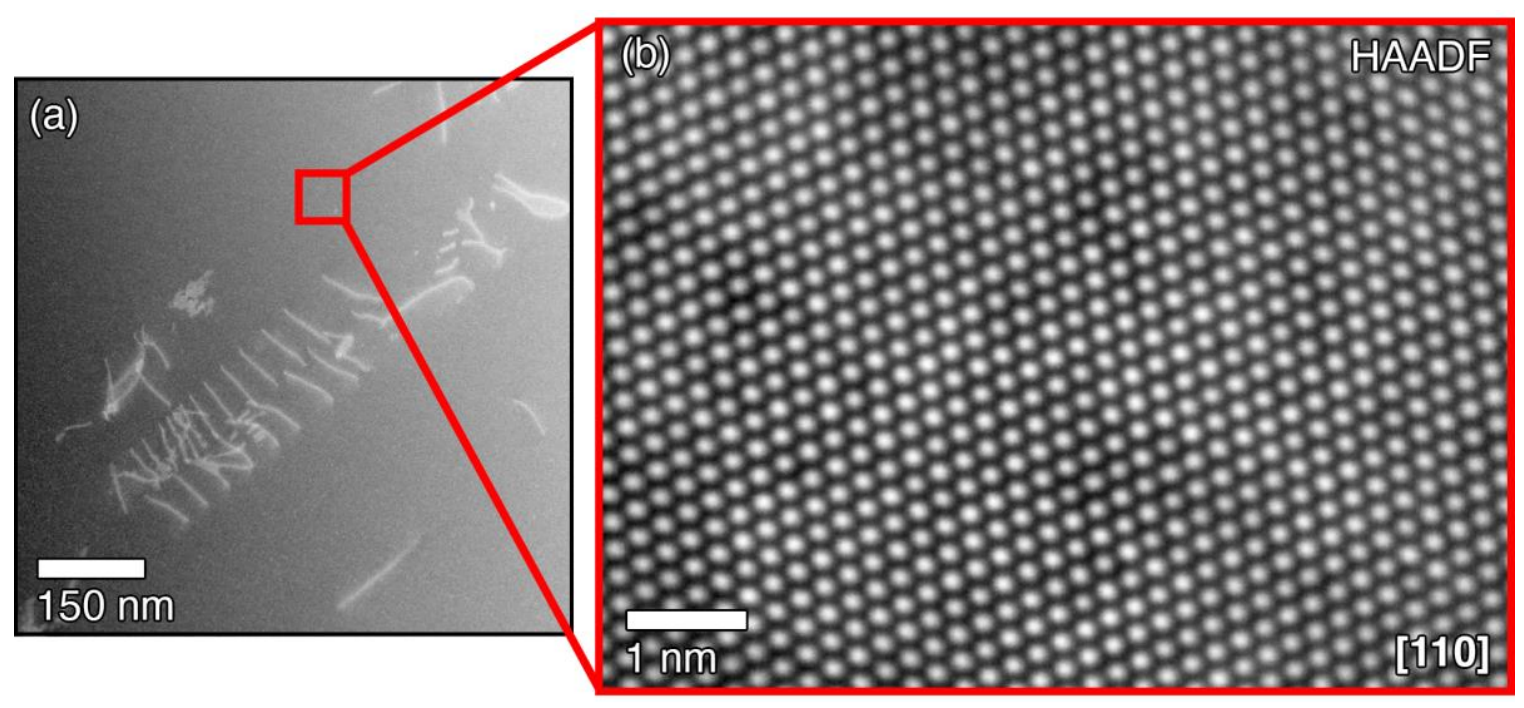


Figure 1. (a) Low-magnification LAADF STEM image of dislocations in the FeMnAlC alloy and (b) atomicresolution HAADF RevSTEM image of atom columns along the [110] zone axis.
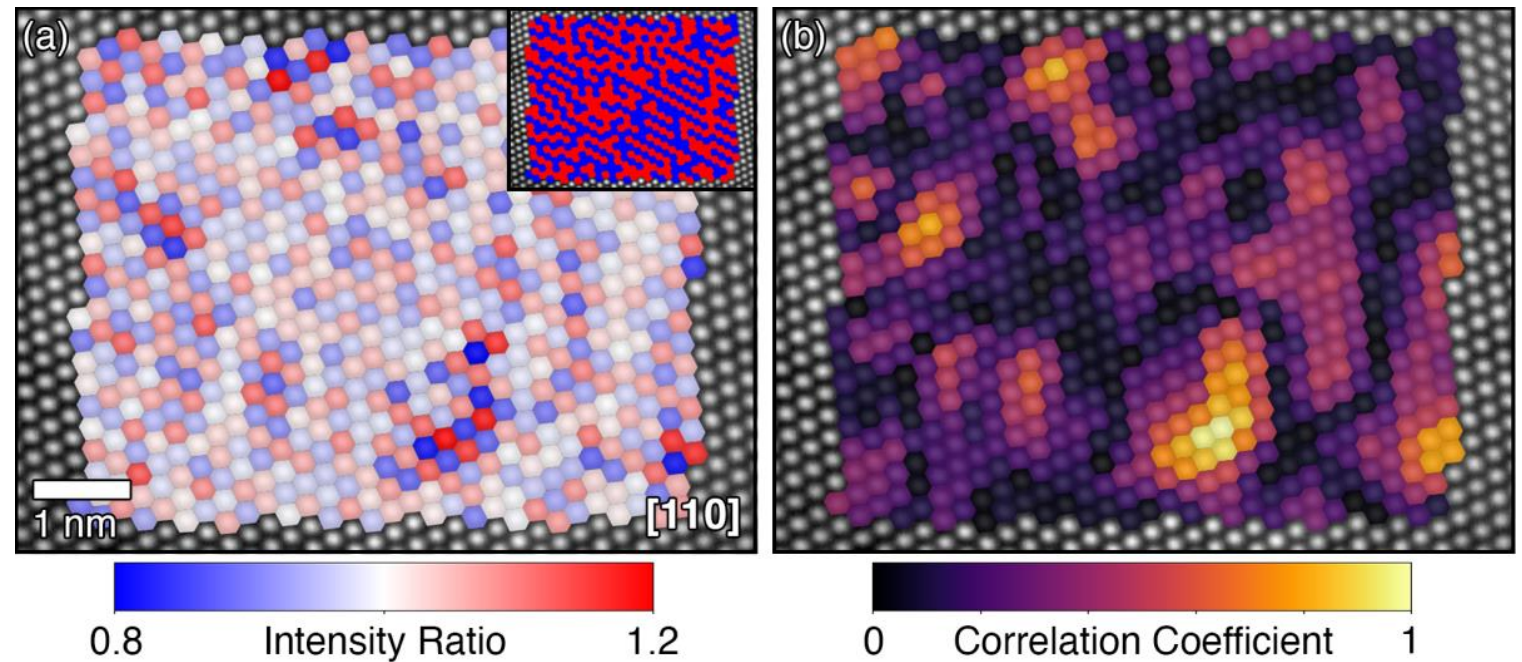

Figure 2. (a) Post-analysis HAADF STEM image of the normalized atom column intensity ratios. The inset shows a binary intensity deviation from the mean in which ordering and anti-phase boundaries are visible. (b) HAADF STEM image overlaid with the correlated intensity ratios from (a), where regions of increasing ordering correspond to a correlation coefficient of 1 .

\section{References}

[1] H. Kim et al., Sci. Technol. Adv. Mater. 14 (2013), 014205.

[2] J. D. Yoo et al., Mater. Sci. Eng. A 508 (2009), p. 234-240.

[3] V. Gerold and H.P. Karnthaler, Acta Metall. 37 (1989), p. 2177-83.

[4] C. Niu et al., Appl. Phys. Lett. 106 (2015), 161906.

[5] S.-D. Kim et al., Sci. Rep. 9 (2019), 15171.

[6] X. Sang and J. M. LeBeau, Ultramicroscopy 138 (2014), p. 28.

[7] We acknowledge support for this work from the National Science Foundation (CMMI-1922206). We also thank Hyunseok Oh and C. Cem Tasan for providing the FeMnAlC sample. 